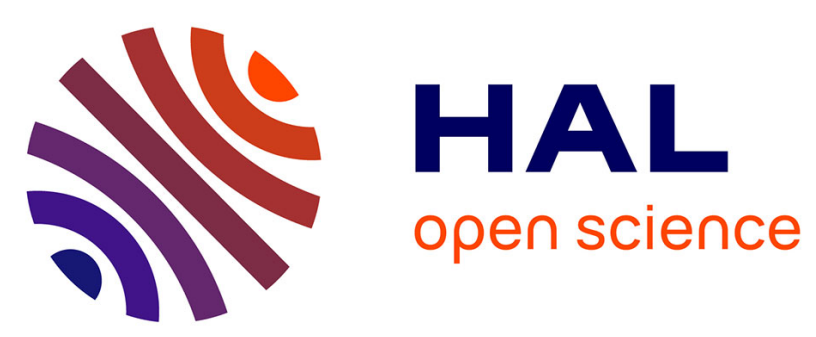

\title{
Modèles à effets aléatoires pour l'analyse longitudinale de la qualité de vie relative à la santé dans l'essai randomisé PRODIGE 4/ACCORD 11
}

Antoine Barbieri, Thierry Conroy, Sophie Gourgou-Bourgade, Amélie Anota, Franck Bonnetain, Christian Lavergne, Caroline Bascoul-Mollevi

\section{To cite this version:}

Antoine Barbieri, Thierry Conroy, Sophie Gourgou-Bourgade, Amélie Anota, Franck Bonnetain, et al.. Modèles à effets aléatoires pour l'analyse longitudinale de la qualité de vie relative à la santé dans l'essai randomisé PRODIGE 4/ACCORD 11. Epidemiology and Public Health = Revue d'Epidémiologie et de Santé Publique, 2014, Revue d'Epidémiologie et de Santé Publique, 62 (S4), pp.117-118. 10.1016/j.respe.2014.05.012 . hal-01211823

\section{HAL Id: hal-01211823 \\ https://hal.science/hal-01211823}

Submitted on 8 Oct 2015

HAL is a multi-disciplinary open access archive for the deposit and dissemination of scientific research documents, whether they are published or not. The documents may come from teaching and research institutions in France or abroad, or from public or private research centers.
L'archive ouverte pluridisciplinaire HAL, est destinée au dépôt et à la diffusion de documents scientifiques de niveau recherche, publiés ou non, émanant des établissements d'enseignement et de recherche français ou étrangers, des laboratoires publics ou privés. 


\title{
MODELE A EFFETS ALEATOIRES POUR L'ANALYSE LONGITUDINALE DE LA QUALITE DE VIE RELATIVE A LA SANTE DANS L'ESSAI RANDOMISE PRODIGE 4 / ACCORD 11
}

\author{
A Barbieri ${ }^{(1,2)}$, T Conroy ${ }^{(3,4)}$, S Gourgou-Bourgade ${ }^{(1)}$, A Anota $^{(4,5)}$, F Bonnetain $^{(4,5)}$, C \\ Lavergne $^{(2)}$ C Bascoul-Mollevi ${ }^{(1)}$ \\ (1) Unité de Biométrie, Institut régional du Cancer de Montpellier (ICM) - Val d'Aurelle \\ (2) Institut de Mathématiques et de Modélisation de Montpellier (I3M), Université de \\ Montpellier 2 \\ (3) Institut de Cancérologie de Lorraine, Nancy \\ (4) Plateforme nationale Qualité de Vie et Cancer \\ (5) Unité de Méthodologie et de Qualité de Vie en Cancérologie, EA 3181, CHU de \\ Besançon
}

INTRODUCTION La qualité de vie relative à la santé (QdV) est devenue un des objectifs prioritaires des essais cliniques en cancérologie pour évaluer l'efficacité d'une prise en charge. Cependant, l'analyse longitudinale de ce critère reste difficile de par la nature des données. En effet, on observe à la fois des réponses multiples, répétées et ordinales. L'objectif de ce travail est de proposer une méthode alternative pour l'analyse longitudinale de la QdV fondée sur les observations brutes des questionnaires standards développés par l'EORTC. Le modèle a été appliqué aux données de QdV de l'essai PRODIGE 4 / ACCORD11/0102 qui est un essai clinique randomisé mené sur 342 patients traités par FOLFIRINOX ou Gemcitabine pour un cancer du pancréas métastatique.

METHODES La QdV n'est pas directement mesurable et est considérée comme un trait latent accessible par l'intermédiaire des réponses aux items. Différentes théories existent pour l'analyse des dimensions des questionnaires de QdV. On différencie la théorie classique (Classical Test Theory, CTT) de la théorie moderne de réponse à l'item (Item Response Theory, IRT). La CTT se fonde sur les observations d'un score qui correspond à la moyenne pondérée des réponses aux items. Le score qui est considéré comme une variable continue se substitue alors à l'utilisation des observations brutes. Sous ces conditions, le modèle linéaire mixte (L2M) est le plus utilisé pour l'analyse longitudinale de la QdV. Les modèles issus de l'IRT sont proposés comme une alternative au premier. Ces modèles peuvent être vus comme des modèles linéaires mixtes généralisés pour données ordinales et supposent l'existence d'un modèle probabiliste liant les réponses aux items au trait latent représentant la QdV. Pour l'analyse longitudinale, nous avons étendu le modèle PCM proposé par Master (1982) afin de prendre en compte des covariables cliniques et les caractéristiques des données. La réponse cachée continue inobservable suit alors un modèle de régression linéaire incorporant des effets fixes et aléatoires. Les deux modèles proposés ont été implémentés sous le logiciel SAS avec les procédures mixed et nlmixed.

RESULTATS Les modèles L2M et PCM longitudinal (LPCM) ont été utilisés pour analyser la perception de la QdV des patients sur l'ensemble des dimensions du questionnaire QLQC30 afin de comparer l'effet des deux traitements en considérant les évaluations à l'inclusion, à 15 jours, puis à 1, 2, 4, 6, 8 et 10 mois. La perception de la QdV est similaire entre les deux bras de traitement pour les modèles L2M et LPCM. Par contre, son évolution au cours du 
temps est significative pour la fonction émotionnelle, le statut global de santé et les symptômes : fatigue, nausée et vomissement, insomnie, anorexie, constipation. Le niveau de douleur est similaire à l'inclusion et décroit significativement $(\mathrm{p}<0.001)$ au cours du temps. Le modèle LPCM met en évidence une différence d'évolution en fonction du groupe de traitement $(\mathrm{p}=0.03)$. A l'inverse, la perception du symptôme diarrhée présente une différence significative $(\mathrm{p}<.01)$ à l'inclusion mais celle-ci reste stable au cours du temps.

CONCLUSION Nous proposons une approche alternative aux analyses classiques qui prend en compte directement les données brutes recueillies via les questionnaires de QdV. La méthode proposée est prometteuse mais nécessite d'être confirmée sur d'autres essais cliniques dont la QdV est un des critères. 